aware that his conclusions as to the cause of the coagulation have been very ably contested.

The result of this case has been most satisfactory; for the long continued difficulty of articulation led to some little fear that a permanent difficulty of articulation might be the result, as it is said to be in all cases of recovery from embolism of the cerebral arteries.

Cases somewhat similar in character to the one I have detailed are, I am convinced, of much more frequent occurrence previous to and after confinement than are generally supposed; and are reported as, and believed to be simply cases of death from syncope. I have myself seen two cases, both of which proved fatal, occurring in the practice of other medical men; and which, I believe, were cases of embolism; but this disease not being thought of, was not detected, nor was any examination made after death. After great loss of blood, coupled with a feeble state of health, I think it will be well to keep the possibility of such an occurrence in our minds, and to direct such treatment as might tend to prevent it.

The treatment in this case is somewhat novel; as I find that most of the reported cases of embolism have been treated by calomel, croton oil, etc. Such treatment, carried to the extent of acting freely on the bowels, would, in this case, I think, have proved injurious; and in suggesting the use of the carbonate of ammonia, iron, and brandy, both $\mathrm{Mr}$. Ransome and myself were not influenced so much by the general condition of the system; but the special reason for preferring the carbonate of ammonia was founded upon Dr. B. W. Richardson's theory as to its action in maintaining the fluidity of the blood. Whatever may be thought of this theory, I am quite convinced that the line of treatment adopted in this case with such excellent results is the proper treatment for cases of embolism generally; and I should look with little favour upon the administration of calomel, croton oil, etc.

\section{CASE OF POISONING BY KIDNEY-BEANS}

\section{By William Dale, Esq., Ilkeston.}

A FEW days since, $I$ was sent for to see a child five years old, who had been taken suddenly ill a short time previously, and was said to be dying. On my arrival at 8 P.M., I found him bedewed with cold perspiration; the breathing slow and sighing; the pulse at the wrist very rapid and almost imperceptible; the pupils widely dilated, and insensible to light. He could only be roused by speaking loudly, but appeared, when thus roused, to understand what was said. Pressure on the abdomen apparently gave pain, as the child shrank from it, and drew up his legs. The features also expressed suffering.

The history of the case was this. The child had always been remarkably healthy up to the time of this seizure. In the afternoon, he was playing with some kidney-beans. (These beans were some of last year's seeds, which a neighbour had thrown away because they were mildewed, and he thought them unlikely to germinate.) At tea-time, he asked his mother for some water. This almost immediately returned, and with it a quantity of half-digested beans. He then complained of pain in the bowels, and gradually became worse until I saw him. I immediately administered a dessert-spoonful of mustard in warm water; and, vomiting being produced, I ordered copious draughts of warm water, with an occasional teaspoonful of sweet oil. The vomit contained a large quantity of semi-digested kidneybeans. When the vomiting had somewhat abated, I ordered two teaspoonfuls of brandy to be administered occasionally, with a little water. The pulse now became a little stronger, and the breathing more natural; the pupils also began to act slightly, but the drowsiness still remained. He was then ordered to have two teaspoonfuls of castor oil. I also ordered a tablespoonful of strong coffee to be given every halfhour. The castor oil soon produced purging; and from that time amendment commenced. The pulse grew stronger; the breathing more natural; the pupils more active ; and the drowsiness less. In the morning, the only symptom remaining was a little tenderness at the epigastrium.

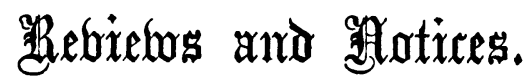

The Science and Art of Surgery: being a Treatise on Surgical Injuries, Diseases, and Operations. By John E. Erichsen, Professor of Surgery and of Clinical Surgery in University College, and Surgeon to University College Hospital. Fourth Edition, enlarged and carefully revised. Illustrated by 517 Engravings on Wood. Pp. 1280. London: 1864.

A System of Surgery. By James Miller, F.R.C.S.E. ; Surgeon in Ordinary to the Queen for Scotland; Professor of Surgery in the University of Edinburgh; etc. Pp. 1387. Edinburgh : 1864.

These two classical works on Surgery have appeared nearly simultaneously in London and Edinburgh; their authors being well known teachers of chirurgical knowledge in their respective localities. Mr. ERICHsen furnishes a fourth edition of his well known work. Mr. Mrller's book is, we believe, the result of the union into one volume, with the necessary emendations, of the two works which have hitherto been published under the titles of the Principles and the Practice of Surgery.

Of two such works, with the favourable antecedents belonging to them, there is little to be said by the reviewer; and any attempt to determine positively which is the better of the two would be, at the least, a difficult task in more senses than one. Yet, in their arrangement, and in some other matters, there is a marked difference between the two books.

Mr. Erichsen arranges his subjects under the three divisions of General Principles, Surgical Injuries, and Surgical Diseases; and in the latter two divisions, after first noticing each injury or disease in its general bearings, he describes the lesions affecting the various parts of the body. Pathological considerations, and the description of necessary operations, are with him incorporated with the description of the injury or disease to which they specially belong. An exception, however, occurs in the case of amputations, which have a chapter to themselves in the works of both authors-Mr. Erichsen placing at near the beginning of his book, while Mr. Miller places it at the end. Mr. Miller places his pathological remarks, and the general descriptions of such diseases as aneurism, fracture, dislocation, etc., in the first part of his book-that corresponding to his Principles of Surgery; while a regular arrangement of diseases and injuries is adopted in the remaining portion. Mr. Erichsen, considering that the diseases of the 